\title{
Total Variation Image Restoration Method Based on Subspace Optimization
}

\author{
XiaoGuang Liu $\mathbb{D}^{1}$ and XingBao Gao $\mathbb{D}^{2}$ \\ ${ }^{1}$ School of Computer Science and Technology, Southwest University for Nationalities, Chengdu, Sichuan 610041, China \\ ${ }^{2}$ College of Mathematics and Information Science, Shaanxi Normal University, Xian 710062, China \\ Correspondence should be addressed to XiaoGuang Liu; dtcr-gg@163.com
}

Received 5 October 2017; Accepted 13 December 2017; Published 21 January 2018

Academic Editor: Daniel Zaldivar

Copyright (c) 2018 XiaoGuang Liu and XingBao Gao. This is an open access article distributed under the Creative Commons Attribution License, which permits unrestricted use, distribution, and reproduction in any medium, provided the original work is properly cited.

\begin{abstract}
The alternating direction method is widely applied in total variation image restoration. However, the search directions of the method are not accurate enough. In this paper, one method based on the subspace optimization is proposed to improve its optimization performance. This method corrects the search directions of primal alternating direction method by using the energy function and a linear combination of the previous search directions. In addition, the convergence of the primal alternating direction method is proven under some weaker conditions. Thus the convergence of the corrected method could be easily obtained since it has same convergence with the primal alternating direction method. Numerical examples are given to show the performance of proposed method finally.
\end{abstract}

\section{Introduction}

Digital image restoration has a wide application in various areas including Navigation, Aerospace, and Biomedicine (see $[1-3]$ and the references therein). In general, the relationship between the original image $\widehat{f} \in R^{p}$ and the observed image $g \in R^{q}$ is

$$
g=A \widehat{f}+b,
$$

where $b \in R^{q}$ is the additive noise and the spatial-invariant matrix $A \in R^{q \times p}$ represents the degradation system caused by problems such as motion blur, distortion radiation, and distortion wavelets in seismic imaging, $p=p_{1} p_{2}$ with $p_{1}$ and $p_{2}$ being the number of rows and columns, respectively, when images are expressed as a matrix. In particular, matrix $A$ represents Block Toeplitz-plus-Hankel matrix (with Toeplitzplus-Hankel blocks) or Block Toeplitz matrix (with Toeplitz blocks) when Neumann boundary condition and the zero boundary condition are used, respectively [4].

The objective of the image restoration is to estimate the original image $\widehat{f}$ according to some a priori knowledge about the degradation system $A$, the additive noise $b$, and the observed image $g$. However, it often tends to be very ill-conditioned when the reverse process of model (1) is only used to get an ideal estimation $f^{*}$. Thus one of the effective ways to solve these problems is to combine some a priori information of the original image and define the regularization solution; that is, $f^{*}$ is a minimizer of the following cost (energy) function:

$$
J(f)=\theta(A f-g)+\alpha \Phi(f),
$$

where $\theta: R^{q} \rightarrow R$ is the measure of the difference between Af and $g$. The regularization term $\Phi$ embodies a priori information and a regularization parameter $\alpha>0$ is used to control the tradeoff between the terms $\theta$ and $\Phi$.

In general, $\theta(x)=\|x\|_{2}^{2}=\sum_{i=1}^{n} x_{i}^{2}$ (the square of $l_{2}$ norm of vector $x \in R^{n}$ ) when the additive noise meets Gaussian distribution. $\theta(x)=\|x\|_{1}=\sum_{i=1}^{n}\left|x_{i}\right|\left(l_{1}\right.$-norm of vector $x \in R^{n}$ ) when the additive noise meets non-Gaussian distributions such as uniform and speckle.

The regularization term $\Phi(f)=\sum_{i \in I} \phi\left(\left\|D_{i} f\right\|_{2}\right)$, where $I=\{1,2, \ldots, p\}, \phi: R \rightarrow R_{+}=\{t \in R: t \geq 0\}$ is the potential function, $D_{i}: R^{p} \rightarrow R^{d}$ is the difference operator which can be seen as a $d \times p$ matrix and used to create the difference 
vector between $i$ th pixel and its $d$ neighboring pixels. Here $D_{i}=\left[D_{i}^{1} ; D_{i}^{2}\right]$ with

$$
\begin{array}{r}
D_{i}^{1}[j]=0 \quad \forall j \in I, \text { if } i \in I_{1}=\left\{p_{2}, 2 p_{2}, \ldots, p_{1} p_{2}\right\}, \\
\begin{array}{r}
D_{i}^{1}[i]=-1, D_{i}^{1}[i+1]=1, D_{i}^{1}[j]=0 \\
\forall j \notin\{i, i+1\}, \text { if } i \notin I_{1},
\end{array} \\
D_{i}^{2}[j]=0 \\
\forall j \in I, \text { if } i \in I_{2}=\left\{\left(p_{1}-1\right) p_{2}+1,\left(p_{1}-1\right) p_{2}+2, \ldots, p_{1} p_{2}\right\}, \\
D_{i}^{2}[i]=-1, D_{i}^{2}\left[i+p_{2}\right]=1, D_{i}^{2}[j]=0 \\
\forall j \notin\left\{i, i+p_{2}\right\}, \text { if } i \notin I_{2} .
\end{array}
$$

In image restoration, the potential function $\phi$ plays a key role so that it is intensively studied in recent decades [5-20]. Two classes of regularization terms are well known. One is the Tikhonov class [5-7], for example, $\Phi(f)=\sum_{i \in I}\left\|D_{i} f\right\|_{2}^{2}$. In these cases, the minimum point of (2) can be easily solved due to differentiability. However, these methods tend to make restored images overly smooth and could not protect the clear boundaries.

The other class is based on total variation (TV) regularization [8]:

$$
\Phi(f)=\|f\|_{\mathrm{TV}}=\sum_{i \in I}\left\|D_{i} f\right\|_{2}=\sum_{i \in I} \sqrt{\left(D_{i}^{1} f\right)^{2}+\left(D_{i}^{2} f\right)^{2}} .
$$

In comparison to the first class, an obvious benefit is that the clear boundaries can be recovered well. Thus many effective methods are proposed to solve the TV deblurring problem (see [8-23]). However, they suffer from some numerical difficulties, since the TV regularization is nondifferentiable. In order to overcome the drawback, in [16], the TV regularization is modified as

$$
\Phi(f)=\|f\|_{\mathrm{TV}, \varepsilon}=\sum_{i \in I} \sqrt{\left(D_{i}^{1} f\right)^{2}+\left(D_{i}^{2} f\right)^{2}+\varepsilon},
$$

where $0<\varepsilon \ll 1$. Obviously, the regularization is differentiable and many classical optimization methods can be applied [24, 25]. Unfortunately, experimental results show that $\varepsilon$ must be small enough to keep the restoration quality, but the efficiency of algorithms will be reduced with smaller $\varepsilon[16,20]$.

By using the following modified TV regularization,

$$
\Phi(f)=\sum_{i \in I}\left|D_{i}^{1} f\right|+\left|D_{i}^{2} f\right|,
$$

a primal-dual active set method is proposed in [17], but it has no rotational invariant [8].

Recently, the alternating direction method [26] is used to solve the $l_{2}$-TV and $l_{1}$-TV image restoration models, and better performance has been obtained [18-23, 27]. It divides original problem into some simple subproblems and solves them alternatively. The downside is that the search directions are not accurate enough which could reduce the performance of algorithms (see the analysis in Section 3).
Based on the considerations above, we will take the algorithms in $[18,20]$ as examples and present a method to improve the performance of the alternating direction method used in $l_{2}$-TV and $l_{1}$-TV models. By means of the subspace optimization [28], it corrects the search direction of the primal alternating direction method by using the energy function and a linear combination of the previous search directions. In addition, the convergence of the primal alternating direction method is proven under some weaker conditions. Thus the convergence of the corrected method could be easily obtained by the equivalence between them. Numerical examples are given to shown the performance of proposed method.

The outline of this paper is as follows. Some preliminaries are stated in Section 2. A $l_{1}$-TV image restoration algorithm based on subspace optimization is proposed in Section 3. The convergence analysis and numerical examples are given in Sections 4 and 5, respectively. Finally, some concluding remarks are given in Section 6.

\section{Preliminaries}

In this section, we first propose some basic definitions and properties; then the alternating direction method to solve $l_{1}$-TV model in [20] will be introduced. The alternating direction method in [18] ( $l_{2}$-TV model) will be omitted here since it is more simpler. But the comparison experimental results of the method and its corrected method still will be listed in Section 5.2. In the following, let $D_{h}$ and $D_{v}$ be the one-side difference matrix on the horizontal direction and vertical direction, respectively, $D=\left(D_{h}, D_{v}\right)^{\top}$, Null $(A)$ is the null space of matric $A, x^{i}$ is the $i$ th iteration, and $x_{(i)}$ is the $i$ th element of vector $x$.

Definition 1 (see [20]). An operator $\mathscr{P}$ is called nonexpansive if for any $x_{1}, x_{2} \in X \subset R^{n}$, we have

$$
\left\|\mathscr{P}\left(x_{1}\right)-\mathscr{P}\left(x_{2}\right)\right\|_{2} \leq\left\|x_{1}-x_{2}\right\|_{2} .
$$

Specially, $\mathscr{P}$ is called $\beta$-averaged nonexpansive if there exists some nonexpansive operator $\mathscr{A}$ and $\beta \in(0,1)$ such that $\mathscr{P}=$ $(1-\beta) \mathscr{I}+\beta \mathscr{A}$, where $\mathscr{I}$ is the identity operator.

Lemma 2 (see [20]). If $\rho>0$, then the minimizer of $\psi(s)=$ $|x-s|^{2}+\rho|s|$ is given by the following:

$$
v(x)= \begin{cases}x-\frac{\rho}{2}, & x>\frac{\rho}{2}, \\ 0, & |x| \leq \frac{\rho}{2}, \\ x+\frac{\rho}{2}, & x<-\frac{\rho}{2} .\end{cases}
$$

We know from [20] that the operator $\nu(x)$ is nonexpansive.

Lemma 3 (see [29]). Let $\varphi$ be convex and semicontinuous, $\beta>$ 0 and

$$
\widehat{x}=\arg \min _{x}\|y-x\|_{2}^{2}+\beta \varphi(x) .
$$

Define $\mathcal{S}$ such that $\hat{x}=\mathcal{S}(y)$ for each $y$; then $\mathcal{S}$ is $(1 / 2)$ averaged nonexpansive. 
Definition 4 (see [25]). A function $\varphi: R^{n} \rightarrow R(\mathrm{i})$ is said to be proper over a set $X \subset R^{n}$ if $\varphi(x)<+\infty$ for at least one $x \in X$ and $\varphi(x)>-\infty$ for all $x \in X$ and (ii) is said to be coercive over a set $X \subset R^{n}$ if for every sequence $\left\{x_{k}\right\} \subset X$ such that $\left\|x_{k}\right\|_{2} \rightarrow \infty$ we have

$$
\lim _{k \rightarrow \infty} \varphi\left(x_{k}\right)=\infty
$$

When $X=R^{n}$, we say that $\varphi$ is coercive on $R^{n}$.

Lemma 5 (see [30]). Let $\varphi: R^{n} \rightarrow R$ be a closed, proper, and coercive function. Then the set of minima of $\varphi$ over $R^{n}$ is nonempty and compact.

In [20], the $l_{1}-T V$ image restoration model is

$$
\arg \min _{f}\|A f-g\|_{1}+\alpha\|f\|_{T V} \text {. }
$$

To avoid the numerical difficulties caused by the nondifferentiability, two auxiliary variables $\omega \in R^{q}$ and $\eta \in R^{p}$ are used to cope with the nonsmooth terms $\|A f-g\|_{1}$ and $\|f\|_{T V}$. Then (12) has been transformed as

$$
\begin{aligned}
\arg \min _{f, \omega, \eta} F(f, \omega, \eta)= & \|A f-\omega\|_{2}^{2}+\alpha\|\eta\|_{T V} \\
& +\alpha_{1}\|\omega-g\|_{1}+\alpha_{2}\|\eta-f\|_{2}^{2},
\end{aligned}
$$

where the parameters $\alpha_{1}>0$ and $\alpha_{2}>0$ are used to ensure the closeness of $g$ and $\omega, f$ and $\eta$, respectively. When applied to (13), the alternating direction method is

$$
\begin{aligned}
\mathscr{H}_{A}\left(\omega^{i}, \eta^{i}\right) & :=f^{i+1} \\
& =\arg \min _{f}\left\|A f-\omega^{i}\right\|_{2}^{2}+\alpha_{2}\left\|\eta^{i}-f\right\|_{2}^{2}, \\
\mathscr{H}_{l_{1}}\left(f^{i+1}\right) & :=\omega^{i+1} \\
& =\arg \min _{\omega}\left\|A f^{i+1}-\omega\right\|_{2}^{2}+\alpha 1\|\omega-g\|_{1}, \\
\mathscr{H}_{T V}\left(f^{i+1}\right) & :=\eta^{i+1} \\
& =\arg \min _{\eta} \alpha_{2}\left\|\eta-f^{i+1}\right\|_{2}^{2}+\alpha\|\eta\|_{T V} .
\end{aligned}
$$

The first step in (14) is equivalent to solving the nonsingular linear system:

$$
\left(A^{\top} A+\alpha_{2} I\right) f=A^{\top} \omega^{i}+\alpha_{2} \eta^{i} .
$$

It can be solved directly; that is,

$$
f=\left(A^{\top} A+\alpha_{2} I\right)^{-1}\left(A^{\top} \omega^{i}+\alpha_{2} \eta^{i}\right) .
$$

To let computational cost be low, many classical optimization and numerical methods such as CG method and preconditioned iteration methods can be used to solve it [24, 25, 31]. As in $[4,18]$, we suppose that the Neumann boundary conditions are used and the blurring function is symmetric in this paper; then $A$ is a (block) Toeplitz-plus-Hankel matrix and it could be diagonalized by a discrete cosine transform matrix. Thus we could solve the nonsingular linear system utilizing lower cost, since the inverse of blurring matrix A can be computed by using fast cosine transforms; please see [4, 18, 27] for more details.
Since

$$
\begin{aligned}
\left\|A f^{i+1}-\omega\right\|_{2}^{2}+\alpha_{1}\|\omega-g\|_{1} \\
\quad=\sum_{k=1}^{q}\left\{\left(\left(A f^{i+1}\right)_{(k)}-\omega_{(k)}\right)^{2}+\alpha_{1}\left|\omega_{(k)}-g_{(k)}\right|\right\},
\end{aligned}
$$

the second step in (14) is equivalent to solving the minimizers of $q$ functions in the form of $\nu(s)=|x-s|^{2}+\alpha_{1}|s|$. We can know from Lemma 2 that the $k$ th element of its solution $\omega^{i+1}$ is

$$
\omega_{(k)}^{i+1}= \begin{cases}\left(A f^{i+1}\right)_{(k)}-\frac{\alpha_{1}}{2}, & \left(A f^{i+1}-g\right)_{(k)}>\frac{\alpha_{1}}{2}, \\ g_{(k)}, & \left|\left(A f^{i+1}-g\right)_{(k)}\right| \leq \frac{\alpha_{1}}{2}, \\ \left(A f^{i+1}\right)_{(k)}+\frac{\alpha_{1}}{2}, & \left(A f^{i+1}-g\right)_{(k)}<-\frac{\alpha_{1}}{2} .\end{cases}
$$

The third step in (14) is a $l_{2}$-TV model with the blurring matrix $A$ is the identity matrix, that is, image denoising problem. Many methods can solve it effectively [13-15], and Chambolle' projection algorithm [13] is used in [20].

\section{The Method Based on Subspace Optimization}

Based on the principle of alternating direction method above, it is easy to know that the solution $l^{i+1}=\left[f^{i+1} ; \omega^{i+1} ; \eta^{i+1}\right]$ of (14) is different from the solution of (13), so the performance of solving the minima of (13) may be lowered. Thus a method based on subspace optimization [28] will be proposed to improve the optimization performance of (14) in this section.

Firstly, we introduce subspace optimization method. Let $\bar{l}^{i+1}=\left[\bar{f}^{i+1} ; \bar{\omega}^{i+1} ; \bar{\eta}^{i+1}\right]$ denote the solution corrected by subspace optimization method, and

$$
S^{i+1}=\left[t_{0}^{i+1}, t_{1}^{i+1}, \ldots, t_{m}^{i+1}\right]
$$

where $t_{0}^{i+1}=l^{i+1}-\bar{l}^{i}$ and $t_{k}^{i+1}=\bar{l}^{i+1-k}-\bar{l}^{i-k}(1 \leq k \leq m \leq i)$, then

$$
\begin{aligned}
\bar{l}^{i+1} & =\bar{l}^{i}+S^{i+1} \pi^{i+1}, \\
\pi^{i+1} & =\arg \min _{\pi} F\left(\bar{l}^{i}+S^{i+1} \pi\right) .
\end{aligned}
$$

Supposing $\pi^{i+1} \in R^{m+1}$ is selected as $(1,0, \ldots, 0)$ in (20), then the equation $\bar{l}^{i+1}=l^{i+1}$ is valid. In fact, we can know from (21) that $\pi^{i+1}$ is a minimum point of the cost function: $F\left(\pi^{i+1}\right)=F\left(\bar{l}^{i}+S^{i+1} \pi\right)$; thus $F\left(\bar{l}^{i+1}\right) \leq F\left(l^{i+1}\right)$ must be true. It is helpful to improve the performance of original alternating direction method on every iteration, and the key problem is how to solve (21) now. To reduce the computational cost, let $S^{i+1}=\left[t_{0}^{i+1}\right]$ in this paper.

Next, the correction method of (14) will be proposed according to three cases. 
Case $1\left(\omega_{(i)} \neq g_{(i)}\right.$ and $D_{i}^{1} \eta \neq 0$ or $D_{i}^{2} \eta \neq 0$ for $\left.\forall i \in I\right)$. Obviously, the function $F(f, \omega, \eta)$ is smooth; we have

$$
\nabla F=\left(\begin{array}{c}
2 A^{\top}(A f-\omega)+2 \alpha_{2}(f-\eta) \\
2(\omega-A f)+\alpha_{1} \sum_{i \in I} \frac{\omega_{(i)}-g_{(i)}}{\left|\omega_{(i)}-g_{(i)}\right|} e_{i} \\
2 \alpha_{2}(\eta-f)+\alpha \sum_{i \in I}^{-1 / 2} x_{i} z_{i}^{\top}
\end{array}\right),
$$

$\nabla^{2} F$

$$
=\left(\begin{array}{ccc}
2 A^{\top} A+2 \alpha_{2} I & -2 A^{\top} & -2 \alpha_{2} I \\
-2 A & 2 I & 0 \\
-2 \alpha_{2} I & 0 & 2 \alpha_{2} I+\frac{\partial^{2}\|\eta\|_{\mathrm{TV}}}{\partial \eta^{2}}
\end{array}\right),
$$

where $e_{i}$ denotes the unit column vector with its $i$ th element being 1 and the others being zero, $x_{i}=\left(D_{i}^{1} \eta\right)^{2}+\left(D_{i}^{2} \eta\right)^{2}$, $y_{i}=D_{i}^{1} \eta+D_{i}^{2} \eta$, and $z_{i}=D_{i}^{1}+D_{i}^{2}$. It is expensive to compute $\partial^{2}\|\eta\|_{\mathrm{TV}} / \partial \eta^{2}$, so we let $\partial^{2}\|\eta\|_{\mathrm{TV}} / \partial \eta^{2}=0$ to improve the efficiency of the algorithm because $\nabla^{2} F$ is independent of variable $l$ now.

On the other hand, since $\pi^{i+1}$ is an optimum of the convex function $F$, we can know from (21) and Taylor series expansion that

$$
\begin{aligned}
\left(S^{i+1}\right)^{\top} \nabla F\left(\bar{l}^{i}+S^{i+1} \pi^{i+1}\right) & =0, \\
\nabla F\left(\bar{l}^{i}+S^{i+1} \pi^{i+1}\right) & =\nabla F\left(\bar{l}^{i}\right)+\nabla^{2} F S^{i+1} \pi^{i+1} .
\end{aligned}
$$

Equation (23) implies that

$$
\left(S^{i+1}\right)^{\top}\left(\nabla F\left(\bar{l}^{i}\right)+\nabla^{2} F S^{i+1} \pi^{i+1}\right)=0 .
$$

Obviously, vector $S^{i+1} \neq 0$; then

$$
\begin{aligned}
& \frac{1}{2}\left(S^{i+1}\right)^{\top} \nabla^{2} F S^{i+1}=\left\|A\left(f^{i+1}-\bar{f}^{i}\right)\right\|^{2}+\left\|\omega^{i+1}-\bar{\omega}^{i}\right\|^{2} \\
& -2\left(\omega^{i+1}-\bar{\omega}^{i}\right)^{\top} A\left(f^{i+1}-\bar{f}^{i}\right)+\alpha_{2}\left[\left\|f^{i+1}-\bar{f}^{i}\right\|^{2}\right. \\
& \left.+\left\|\eta^{i+1}-\bar{\eta}^{i}\right\|^{2}-2\left(\eta^{i+1}-\bar{\eta}^{i}\right)^{\top}\left(f^{i+1}-\bar{f}^{i}\right)\right] \\
& =\left\|A\left(f^{i+1}-\bar{f}^{i}\right)-\omega^{i+1}+\bar{\omega}^{i}\right\|_{2}^{2}+\alpha_{2} \| f^{i+1}-\bar{f}^{i} \\
& -\eta^{i+1}+\bar{\eta}^{i} \|_{2}^{2} \geq 0 .
\end{aligned}
$$

Thus $\left(S^{i+1}\right)^{\top} \nabla^{2} F S^{i+1}=0 \Leftrightarrow\left\|A\left(f^{i+1}-\bar{f}^{i}\right)-\omega^{i+1}+\bar{\omega}^{i}\right\|_{2}^{2}+$ $\alpha_{2}\left\|f^{i+1}-\bar{f}^{i}-\eta^{i+1}+\bar{\eta}^{i}\right\|_{2}^{2}=0 \Leftrightarrow A\left(f^{i+1}-\bar{f}^{i}\right)=\omega^{i+1}-\bar{\omega}^{i}$ and $f^{i+1}-\bar{f}^{i}=\eta^{i+1}-\bar{\eta}^{i}$. The two equalities do not hold in image restoration since the dimension of vector $f$ is very huge. Therefore $\left(S^{i+1}\right)^{\top} \nabla^{2} F S^{i+1}>0$, and

$$
\pi^{i+1}=-\left(\left(S^{i+1}\right)^{\top} \nabla^{2} F S^{i+1}\right)^{-1}\left(\left(S^{i+1}\right)^{\top} \nabla F\left(\bar{l}^{i}\right)\right)
$$

from (24).

Case $2\left(I_{3}=\left\{i \mid D_{i}^{1} \eta=D_{i}^{2} \eta=0, i \in I\right\}\right.$ is nonempty). Now, $\|\eta\|_{\mathrm{TV}}$ is nonsmooth. Let

$$
\begin{aligned}
\|\eta\|_{\mathrm{TV}+\varepsilon}= & \sum_{i \in I_{3}}\left(\left(D_{i}^{1} \eta\right)^{2}+\left(D_{i}^{2} \eta\right)^{2}+\varepsilon\right)^{1 / 2} \\
& +\sum_{i \in I / I_{3}}\left(\left(D_{i}^{1} \eta\right)^{2}+\left(D_{i}^{2} \eta\right)^{2}\right)^{1 / 2},
\end{aligned}
$$

then $\lim _{\varepsilon \rightarrow 0}\|\eta\|_{\mathrm{TV}+\varepsilon}=\|\eta\|_{\mathrm{TV}}$ and $\partial\|\eta\|_{\mathrm{TV}+\varepsilon} / \partial \eta_{(i)}=\left(x_{i}+\right.$ $\varepsilon)^{-1 / 2} y_{i} z_{i}^{\top}=0\left(y_{i}=0\right)$ when $i \in I_{3}$. Thus $\partial\|\eta\|_{\mathrm{TV}} / \partial \eta_{(i)}=0$ is set when $i \in I_{3}$.

Case $3\left(\exists i \in I\right.$ such that $\left.\omega_{(i)}=g_{(i)}\right)$. The term $\alpha_{1}\left\|\omega_{(i)}-g_{(i)}\right\|_{1}$ is not differential at this case; the Huber function is often used to replace it [25]. For simplicity, $\partial\|\omega-g\|_{1} / \partial \omega_{(i)}=0$ is selected when $\omega_{(i)}=g_{(i)}$.

In summary, the proposed algorithm is as follows.

\section{Algorithm}

Step 1. Initialize $\bar{l}^{0}$.

Step 2. Solve

(i) $f^{i+1}=\arg \min _{f}\left\|A f-\omega^{i}\right\|_{2}^{2}+\alpha_{2}\left\|\eta^{i}-f\right\|_{2}^{2}$,

(ii) $\omega^{i+1}=\arg \min _{\omega}\left\|A f^{i+1}-\omega\right\|_{2}^{2}+\alpha_{1}\|\omega-g\|_{1}$,

(iii) $\eta^{i+1}=\arg \min _{\eta} \alpha_{2}\left\|\eta-f^{i+1}\right\|_{2}^{2}+\alpha\|\eta\|_{\mathrm{TV}}$.

Step 3. Compute $\pi^{i+1}$ using (26), and let $\bar{l}^{i+1}=\bar{l}^{i}+S^{i+1} \pi^{i+1}$. Step 4. If $\left\|f^{i+1}-f^{i}\right\|_{2} /\left\|f^{i+1}\right\|_{2}<10^{-4}$, then stop; otherwise go to Step 2 .

\section{Convergence Analysis}

As known from (20) and (26), when $\lim _{i \rightarrow \infty}\left\|t_{0}^{i}\right\|_{2}=0$ and one of the sequences $\left\{l^{i}\right\}$ and $\left\{\bar{l}^{i}\right\}$ is convergent, then the other sequence is also convergent, where $t_{0}^{i}=l^{i}-\bar{l}^{i-1}$. Thus a simple proof of the convergence of algorithm (14) will be given below without the condition that $\mathscr{H}_{l_{1}}\left(\mathscr{H}_{A}(\cdot, \eta)\right)$ and $\mathscr{H}_{\mathrm{TV}}\left(\mathscr{H}_{A}(\omega, \cdot)\right)$ are asymptotically regular which is needed in [20], where

$$
\begin{aligned}
\omega^{i+1} & =\mathscr{H}_{l_{1}}\left(f^{i+1}\right)=\mathscr{H}_{l_{1}}\left(\mathscr{H}_{A}\left(\omega^{i}, \eta^{i}\right)\right) \\
& =\mathscr{T}_{1}\left(\omega^{i}, \eta^{i}\right),
\end{aligned}
$$




$$
\begin{aligned}
\eta^{i+1} & =\mathscr{H}_{\mathrm{TV}}\left(f^{i+1}\right)=\mathscr{H}_{\mathrm{TV}}\left(\mathscr{H}_{A}\left(\omega^{i}, \eta^{i}\right)\right) \\
& =\mathscr{T}_{2}\left(\omega^{i}, \eta^{i}\right), \\
\mathscr{T}_{1}(\cdot) & =\mathscr{H}_{l_{1}}\left(\mathscr{H}_{A}(\cdot, \eta)\right), \\
\mathscr{T}_{2}(\cdot) & =\mathscr{H}_{\mathrm{TV}}\left(\mathscr{H}_{A}(\omega, \cdot)\right) .
\end{aligned}
$$

Lemmas 6 and 7 below appeared in $[18,20]$, but the proofs given by us are simpler.

Lemma 6. Suppose $\operatorname{Null}(A) \cap \operatorname{Null}(D)=\{0\}$; then the sets of fixed points of operators $\mathscr{T}_{1}$ and $\mathscr{T}_{2}$ are nonempty, respectively.

Proof. It is easy to see that the objection function $F$ in (13) satisfies the conditions of Lemma $5[18,20]$. Then $F$ has at least one minimizer $(\bar{f}, \bar{\omega}, \bar{\eta})$ that cannot be decreased by the alternating scheme (14). Thus

$$
\begin{aligned}
& \bar{f}=\mathscr{H}_{A}(\bar{\omega}, \bar{\eta})=\arg \min F(\cdot, \bar{\omega}, \bar{\eta}), \\
& \bar{\omega}=\mathscr{H}_{l_{1}}(\bar{f})=\arg \min F(\bar{f}, \cdot \bar{\eta}), \\
& \bar{\eta}=\mathscr{H}_{\mathrm{TV}}(\bar{f})=\arg \min F(\bar{f}, \bar{\omega}, \cdot), \\
& \bar{\omega}=\mathscr{H}_{l_{1}}\left(\mathscr{H}_{A}(\bar{\omega}, \bar{\eta})\right)=\mathscr{T}_{1}(\bar{\omega}), \\
& \bar{\eta}=\mathscr{H}_{\mathrm{TV}}\left(\mathscr{H}_{A}(\bar{\omega}, \bar{\eta})\right)=\mathscr{T}_{2}(\bar{\eta}) .
\end{aligned}
$$

Therefore $\bar{\omega}, \bar{\eta}$ are the fixed points of $\mathscr{T}_{1}$ and $\mathscr{T}_{2}$, respectively.

Lemma 7. The operators $\mathscr{T}_{1}$ and $\mathscr{T}_{2}$ are nonexpansive.

Proof. From Lemmas 2 and 3, we have

$$
\begin{aligned}
& \left\|\mathscr{T}_{1} \omega_{1}-\mathscr{T}_{1} \omega_{2}\right\|_{2}=\| \mathscr{H}_{l_{1}}\left(\mathscr{H}_{A}\left(\omega_{1}, \eta\right)\right) \\
& -\mathscr{H}_{l_{1}}\left(\mathscr{H}_{A}\left(\omega_{2}, \eta\right)\right)\left\|_{2}=\right\| \psi\left(A \mathscr{H}_{A}\left(\omega_{1}, \eta\right)\right) \\
& -\psi\left(A \mathscr{H}_{A}\left(\omega_{2}, \eta\right)\right)\left\|_{2} \leq\right\| A \mathscr{H}_{A}\left(\omega_{1}, \eta\right) \\
& -A \mathscr{H}_{A}\left(\omega_{2}, \eta\right) \|_{2} \\
& =\| A\left(A^{\top} A+\alpha_{2} I\right)^{-1}\left(A^{\top} \omega_{1}+\alpha_{2} \eta\right) \\
& -A\left(A^{\top} A+\alpha_{2} I\right)^{-1}\left(A^{\top} \omega_{2}+\alpha_{2} \eta\right) \|_{2} \\
& =\left\|A\left(A^{\top} A+\alpha_{2} I\right)^{-1} A^{\top}\left(\omega_{1}-\omega_{2}\right)\right\|_{2} \leq\left\|\omega_{1}-\omega_{2}\right\|_{2},
\end{aligned}
$$

$$
\begin{aligned}
& \left\|\mathscr{T}_{2} \eta_{1}-\mathscr{T}_{2} \eta_{2}\right\|_{2}=\| \mathscr{H}_{\mathrm{TV}}\left(\mathscr{H}_{A}\left(\omega, \eta_{1}\right)\right) \\
& \quad-\mathscr{H}_{\mathrm{TV}}\left(\mathscr{H}_{A}\left(\omega, \eta_{2}\right)\right)\left\|_{2} \leq\right\| \mathscr{H}_{A}\left(\omega, \eta_{1}\right) \\
& -\mathscr{H}_{A}\left(\omega, \eta_{2}\right) \|_{2} \\
& =\| A\left(A^{\top} A+\alpha_{2} I\right)^{-1}\left(A^{\top} \omega+\alpha_{2} \eta_{1}\right) \\
& -A\left(A^{\top} A+\alpha_{2} I\right)^{-1}\left(A^{\top} \omega+\alpha_{2} \eta\right)_{2} \|_{2} \\
& =\left\|A\left(A^{\top} A+\alpha_{2} I\right)^{-1} A^{\top}\left(\eta_{1}-\eta_{2}\right)\right\|_{2} \leq\left\|\eta_{1}-\eta_{2}\right\|_{2} .
\end{aligned}
$$

This completes the proof.

Lemma 8. Let $\left\{\omega^{i}\right\}$ and $\left\{\eta^{i}\right\}$ be generated by (28); then $\sum_{k=1}^{\infty}\left\|\omega^{i_{k}+1}-\omega^{i_{k}}\right\|_{2}^{2}$ and $\sum_{k=1}^{\infty}\left\|\eta^{i_{k}+1}-\eta^{i_{k}}\right\|_{2}^{2}$ are bounded, where $\omega^{i_{k}}$ and $\eta^{i_{k}}$ are the subsequence of $\left\{\omega^{i}\right\}$ and $\left\{\eta^{i}\right\}$, respectively.

Proof. For $F\left(f, \omega^{i_{k}}, \eta^{i_{k}}\right)$ in (13), we have

$$
\begin{aligned}
& F\left(f^{i_{k}}, \omega^{i_{k}}, \eta^{i_{k}}\right)=F\left(f^{i_{k}+1}, \omega^{i_{k}}, \eta^{i_{k}}\right) \\
& \quad+\left(f^{i_{k}}-f^{i_{k}+1}\right)^{\top} \frac{\partial F}{\partial f}\left(f^{i_{k}+1}, \omega^{i_{k}}, \eta^{i_{k}}\right) \\
& \quad+\frac{1}{2}\left(f^{i_{k}}-f^{i_{k}+1}\right)^{\top}\left(A^{\top} A+\alpha_{2} I\right)\left(f^{i_{k}+1}, \omega^{i_{k}}, \eta^{i_{k}}\right) \\
& \quad \cdot\left(f^{i_{k}}-f^{i_{k}+1}\right) .
\end{aligned}
$$

Since $f^{i_{k}+1}$ is the minimizer of $F\left(f, \omega^{i_{k}}, \eta^{i_{k}}\right)$, so

$$
\frac{\partial F}{\partial f}\left(f^{i_{k}+1}, \omega^{i_{k}}, \eta^{i_{k}}\right)=0 .
$$

Then

$$
\begin{gathered}
F\left(f^{i_{k}}, \omega^{i_{k}}, \eta^{i_{k}}\right)-F\left(f^{i_{k}+1}, \omega^{i_{k}}, \eta^{i_{k}}\right) \\
\geq \alpha_{2}\left\|f^{i_{k}+1}-f^{i_{k}}\right\|_{2}^{2} .
\end{gathered}
$$

Notice that $F\left(f^{i_{k}+1}, \omega^{i_{k}+1}, \eta^{i_{k}+1}\right) \leq F\left(f^{i_{k}+1}, \omega^{i_{k}}, \eta^{i_{k}}\right)$, we get

$$
\begin{aligned}
& F\left(f^{i_{k}}, \omega^{i_{k}}, \eta^{i_{k}}\right)-F\left(f^{i_{k}+1}, \omega^{i_{k}+1}, \eta^{i_{k}+1}\right) \\
& \quad \geq \alpha_{2}\left\|f^{i_{k}+1}-f^{i_{k}}\right\|_{2}^{2} .
\end{aligned}
$$

From Lemma 2, $\mathscr{H}_{l_{1}}$ is nonexpansive; that is,

$$
\begin{aligned}
\left\|f^{i_{k}+1}-f^{i_{k}}\right\|_{2}^{2} & \geq\left\|\mathscr{H}_{l_{1}}\left(f^{i_{k}+1}\right)-\mathscr{H}_{l_{1}}\left(f^{i_{k}}\right)\right\|_{2}^{2} \\
& =\left\|\omega^{i_{k}+1}-\omega^{i_{k}}\right\|_{2}^{2},
\end{aligned}
$$




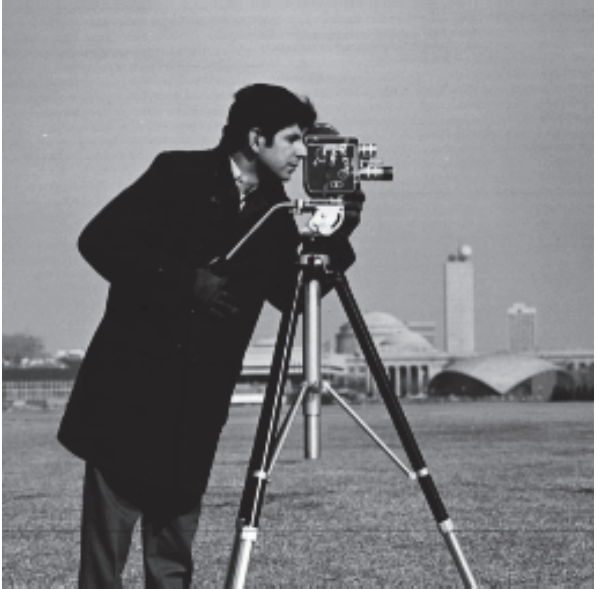

(a)

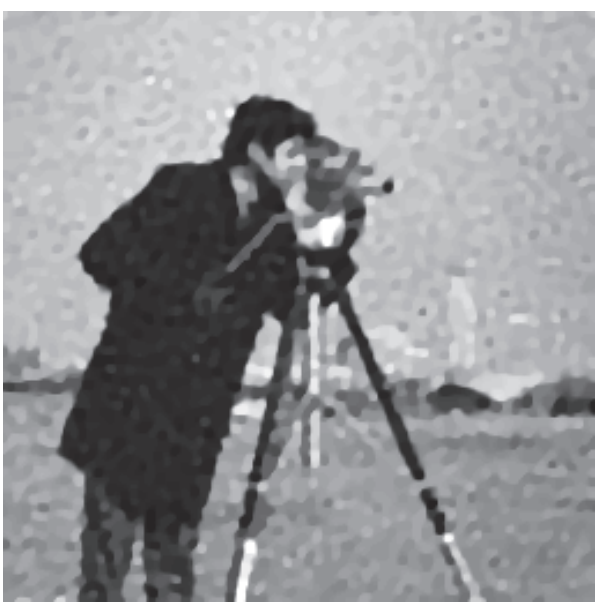

(c)

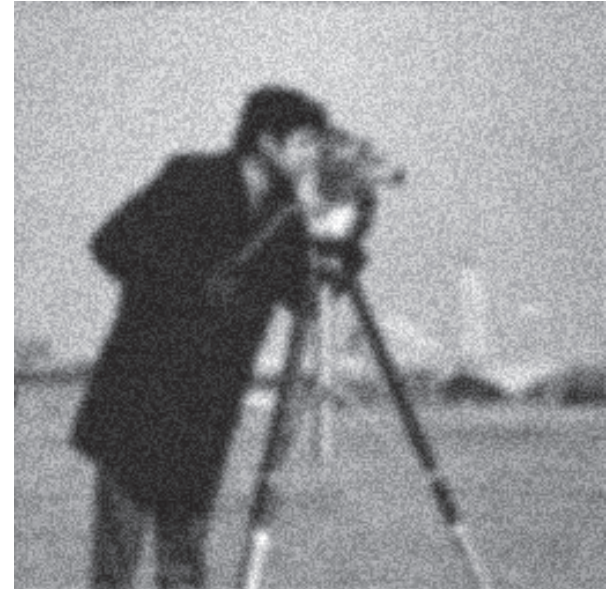

(b)

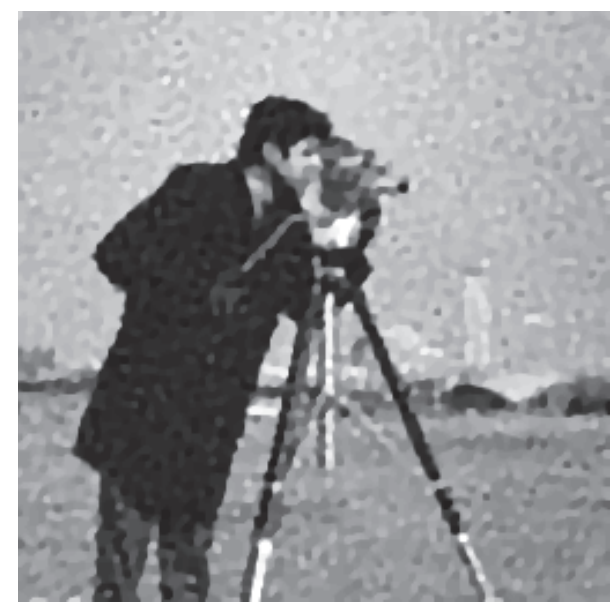

(d)

FIGURE 1: The restored Camera images by different methods with the uniform noise. The random numbers are in the interval $(0,0.2)$, the support being equal to $9 \times 9$. (a) Original image. (b) Observed image. (c) Image restored by algorithm in [20]. (d) Image restored by our method.

and thus

$$
\begin{aligned}
& F\left(f^{i_{k}}, \omega^{i_{k}}, \eta^{i_{k}}\right)-F\left(f^{i_{k}+1}, \omega^{i_{k}+1}, \eta^{i_{k}+1}\right) \\
& \geq \alpha_{2}\left\|\omega^{i_{k}+1}-\omega^{i_{k}}\right\|_{2}^{2} .
\end{aligned}
$$

On the other hand,

$$
F\left(f^{i_{k+1}}, \omega^{i_{k+1}}, \eta^{i_{k+1}}\right) \leq F\left(f^{i_{k}+1}, \omega^{i_{k}+1}, \eta^{i_{k}+1}\right),
$$

and we have

$$
\begin{aligned}
\sum_{k=1}^{\infty} & {\left[F\left(f^{i_{k}}, \omega^{i_{k}}, \eta^{i_{k}}\right)-F\left(f^{i_{k}+1}, \omega^{i_{k}+1}, \eta^{i_{k}+1}\right)\right] } \\
& =F\left(f^{i_{1}}, \omega^{i_{1}}, \eta^{i_{1}}\right)+\sum_{k \rightarrow \infty}\left[F\left(f^{i_{k+1}}, \omega^{i_{k+1}}, \eta^{i_{k+1}}\right)\right. \\
& -F\left(f^{i_{k}+1}, \omega^{i_{k}+1}, \eta^{i_{k}+1}\right) \\
& \left.-F\left(f^{i_{k+1}+1}, \omega^{i_{k+1}+1}, \eta^{i_{k+1}+1}\right)\right]<F\left(f^{i_{1}}, \omega^{i_{1}}, \eta^{i_{1}}\right) .
\end{aligned}
$$

Namely, $\sum_{k=1}^{\infty}\left\|\omega^{i_{k}+1}-\omega^{i_{k}}\right\|_{2}^{2}$ is bounded. Similarly, one can verify that $\sum_{k=1}^{\infty}\left\|\eta^{i_{k}+1}-\eta^{i_{k}}\right\|_{2}^{2}$ is also bounded.

Now we state and prove the convergence result of (14).

Theorem 9. Suppose $\operatorname{Null}(A) \cap \operatorname{Null}(D)=\{0\}$; the sequence $\left\{\left(\omega^{i}, \eta^{i}\right)\right\}$ generated by (14) with any initial point $\left(\omega^{0}, \eta^{0}\right)$ converges to a solution $\left(\omega^{*}, \eta^{*}\right)$ of $(13)$.

Proof. From Lemma 6, let $\bar{\omega}$ be any fixed point of $\mathscr{T}_{1}$; then

$$
\begin{aligned}
\left\|\omega^{i}-\bar{\omega}\right\|_{2} & =\left\|\mathscr{T}_{1}\left(\omega^{i-1}\right)-\mathscr{T}_{1}(\bar{\omega})\right\|_{2} \leq\left\|\omega^{i-1}-\bar{\omega}\right\|_{2} \\
& \leq \cdots \leq\left\|\omega^{0}-\bar{\omega}\right\|_{2}
\end{aligned}
$$

from Lemma 7. Thus the sequence $\left\{\omega^{i}\right\}$ is bounded, and there exists a subsequence $\left\{\omega^{i_{k}}\right\}$ with $\lim _{k \rightarrow \infty} \omega^{i_{k}}=\omega^{*}$. On the 


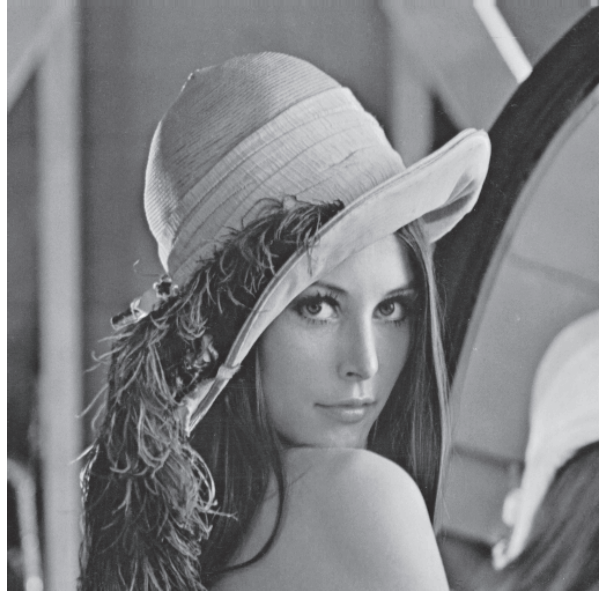

(a)

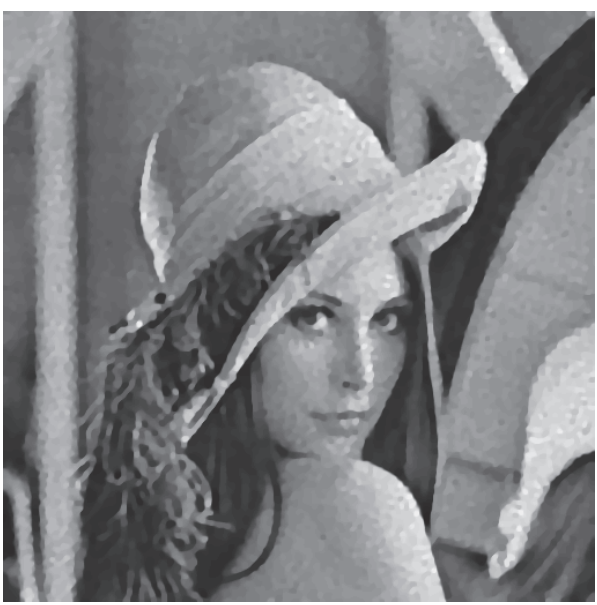

(c)

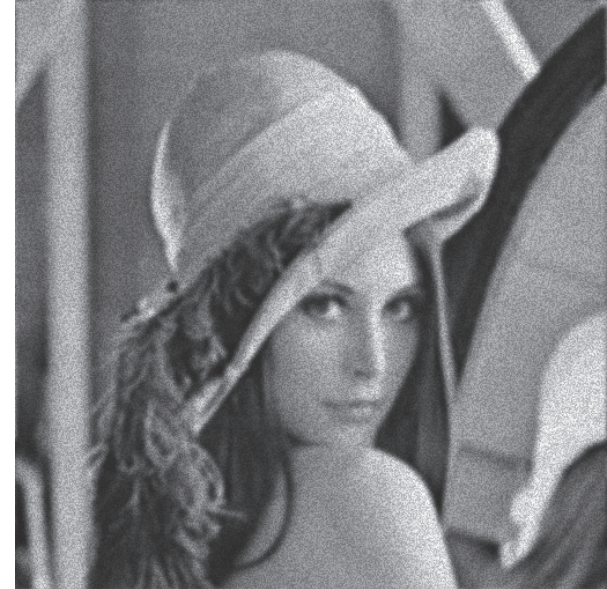

(b)

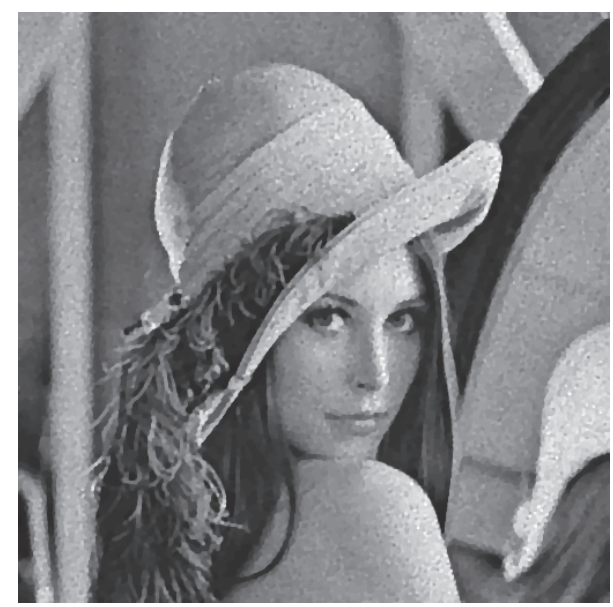

(d)

FIGURE 2: The restored Lena images by different methods with the speckle noise. The noise variance is 0.01 , the support being equal to $7 \times 7$. (a) Original image. (b) Observed image. (c) Image restored by algorithm in [20]. (d) Image restored by our method.

other hand, $\sum_{k=1}^{\infty}\left\|\omega^{i_{k}+1}-\omega^{i_{k}}\right\|_{2}^{2}$ is bounded from Lemma 8; thus

$$
\lim _{k \rightarrow \infty}\left\|\omega^{i_{k}+1}-\omega^{i_{k}}\right\|_{2}^{2}=0
$$

Let $k \rightarrow \infty$, then $\left\|\mathscr{T}_{1}\left(\omega^{*}\right)-\omega^{*}\right\|_{2}=0$; that is, $\omega^{*}$ is also a fixed point of $\mathscr{T}_{1}$. Similarly, to prove (41), one can also verify that the sequence $\left\{\left\|\omega^{i}-\omega^{*}\right\|_{2}\right\}$ is nonincreasing. Thus $\lim _{k \rightarrow \infty} \| \omega^{i}-$ $\omega^{*} \|_{2}=0$ since $\lim _{k \rightarrow \infty} \omega^{i_{k}}=\omega^{*}$.

Following the argument above, one can prove that the sequence $\left\{\eta^{i}\right\}$ generated by (14) with any initial point $\eta^{0}$ converges to a solution $\eta^{*}$ of (13).

Remark 10. If $x \in \operatorname{Null}(D)$, then $x_{(i)}=c$ for all $i \in I$, where $c$ is a nonzero constant. Since matrix $A \geq 0$ in image restoration, so $A x$ is a nonzero vector. It follows that the assumption $\operatorname{Null}(A) \cap \operatorname{Null}(D)=\{0\}$ holds in general.

\section{Experimental Results}

In this section, some numerical results will be provided to show the performance of our method. As in $[18,20]$, the tested images are selected as Cameraman of $256 \times 256$ and Lena of $512 \times 512$. The algorithm in [20] and ours will be compared in Section 5.1 ( $l_{1}$-TV model). The algorithm in [18] and its corrected algorithm will be compared in Section 5.2 $\left(l_{2}\right.$-TV model). All the computational tasks are performed using MATLAB 2016a with Core(TM)2CPU with $2.83 \mathrm{GHz}$ and 3.87 GB of RAM. The average value of ten tests would be selected.

The tested blurring function is chosen to be truncated $2 \mathrm{D}$ Gaussian function:

$$
h(s, t)=\exp \left(\frac{-s^{2}-t^{2}}{2 \sigma^{2}}\right), \quad-3 \leq s, t \leq 3 .
$$

Here three sets of parameters are chosen: (1) the support being equal to $5 \times 5(\sigma=1)$; (2) the support being equal to $7 \times 7(\sigma=1.5) ;(3)$ the support being equal to $9 \times 9(\sigma=2)$. 


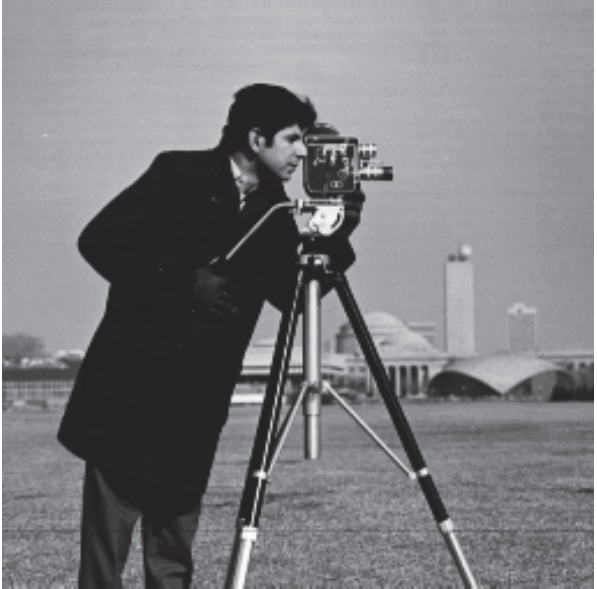

(a)

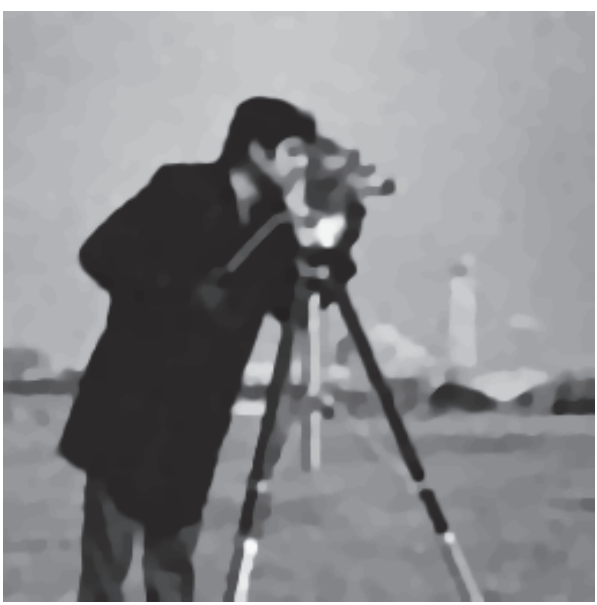

(c)

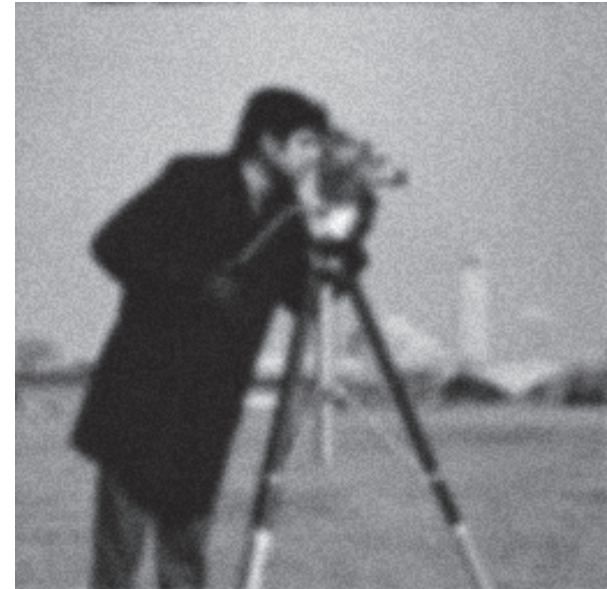

(b)

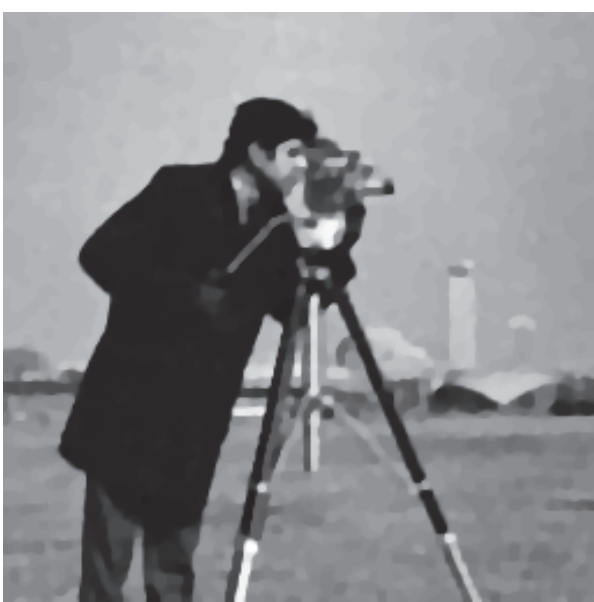

(d)

Figure 3: The restored Camera images by different methods with Gaussian noise. The standard deviation of noise is 0.1 ; the support is $9 \times 9$. (a) Original image. (b) Observed image. (c) Image restored by algorithm in [18]. (d) Image restored by our method.

In all runs, $\mathrm{CPU}$ time is used to compare the efficiency, signal-to-noise ratio (SNR), and peak signal-to-noise ratio (PSNR):

$$
\begin{gathered}
\mathrm{SNR}=20 \log _{10}\left(\frac{\|\hat{f}\|_{2}}{\left\|f^{i+1}-\hat{f}\right\|_{2}}\right), \\
\text { PSNR }=-20 \log _{10}\left(\frac{\left\|f^{i+1}-\widehat{f}\right\|_{2}}{p_{1} p_{2}}\right),
\end{gathered}
$$

which are used to measure the quality of the restored images. The stopping criterion of the algorithms should satisfies

$$
\frac{\left\|f^{i+1}-f^{i}\right\|_{2}}{\left\|f^{i+1}\right\|_{2}} \leq 10^{-4} .
$$

5.1. Comparison Experiment for the $l_{1}-T V$ Model. In this subsection, a comparison of the algorithm in [20] with the proposed method is made under different non-Gaussian additive noises. Firstly, for the uniform noise, we let $\alpha=0.008$, $\alpha_{1}=0.05$, and $\alpha_{2}=0.2$ as in [20]. The uniform random numbers appear in the intervals $(0,0.05),(0,0.1)$, and $(0,0.2)$, respectively. Specially, [20] points out that the noise added to the blur image is independent and identically distributed, so the same set of parameters could be selected for different images when the same kind of noise is considered. This could reduce the computational cost of searching regularization parameters. For the speckle noise, as in [20, 23], let $\alpha \equiv 1$, $\alpha_{1}=2.5 \times 10^{4}$, the continuation scheme about $\alpha_{2}$ will be used, easier subsequences with smaller $\alpha_{2}$ can be solved quickly, and the later subproblems can also be solved relatively quickly with warm starts from previous solutions. The noise variance will be selected as $0,0.01$, and 0.05 respectively. To be fair, the same methods will be used to solve the primal alternating direction method in this paper.

Now, we select two cases that have significant improvement of the visual sense to illustrate our method's restoration quality. Under different conditions, more image restoration 


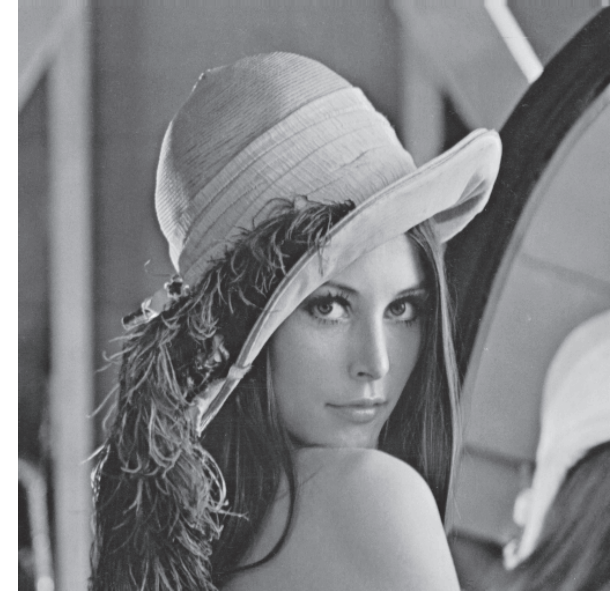

(a)

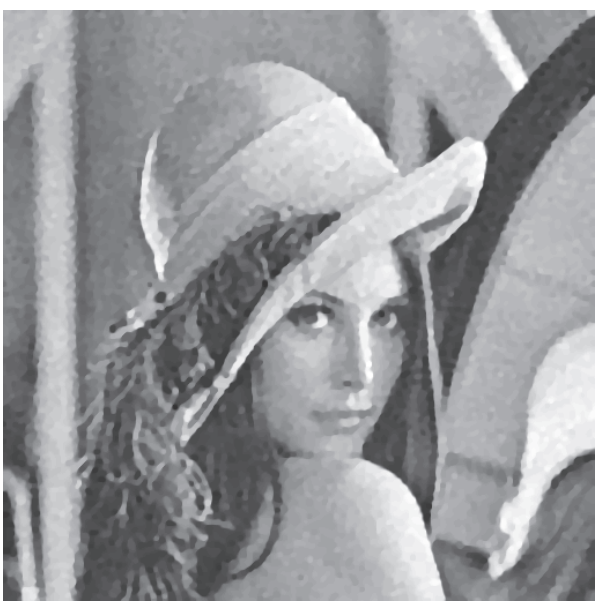

(c)

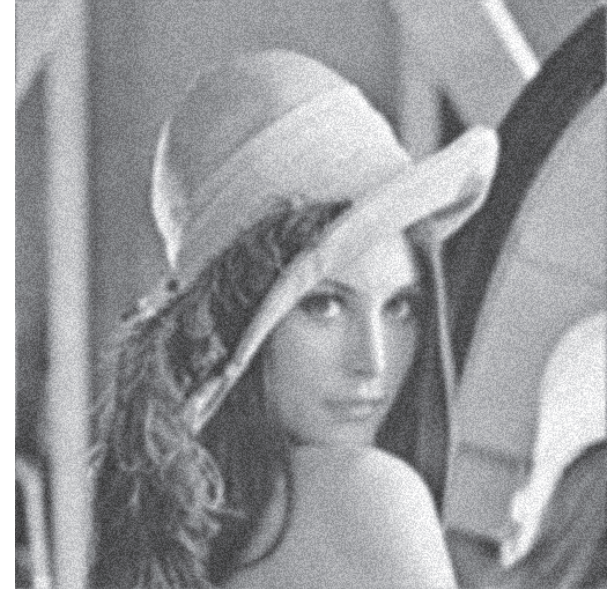

(b)

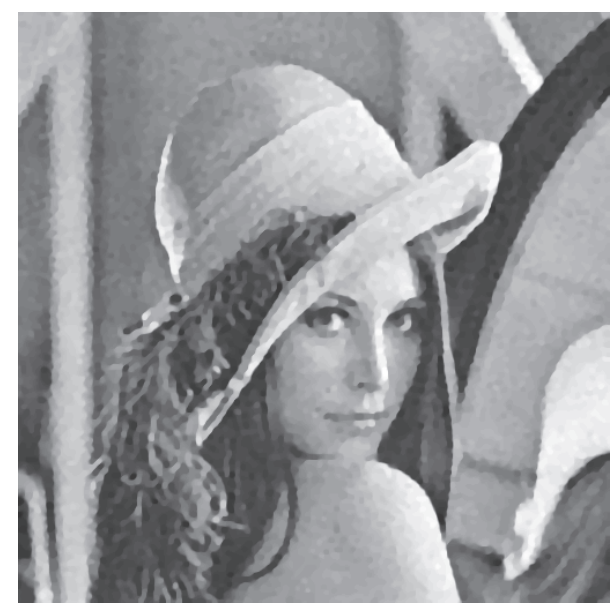

(d)

FIGURE 4: The restored Lena images by different methods with Gaussian noise. The standard deviation of noise is 0.2 ; the support is $9 \times 9$. (a) Original image. (b) Observed image. (c) Image restored by algorithm in [18]. (d) Image restored by our method.

results with uniform noise and speckle noise will be summarized in Tables 1 and 2, respectively. Figure 1(a) shows the original Camera image. Figure 1(b) shows the observed Camera image, where the noise is the uniform noise, the random numbers are in the interval $(0,0.2)$, and the support is equal to $9 \times 9$. Figure $1(\mathrm{c})$ shows the restored Camera image by the algorithm in [20]. Figure 1(d) shows the restored Camera image by our method. Figure 2(a) shows the original Lena image. Figure 2(b) shows the observed Lena image, where the noise is the speckle noise, the noise variance is 0.01 , the support is equal to $7 \times 7$. Figure 2 (c) shows the restored Lena image by the algorithm in [20]. Figure 2(d) shows the restored Lena image by our method.

From Figures 1 and 2, we see that the quality of images restored by our method is improved obviously compared to those restored by the method of [20] in the two cases. For the uniform noise and speckle noise, Tables 1 and 2 show that the SNR and PSNR of the images restored by our method both are higher than the results of [20] in most cases. For the same requirement of relative error, our proposed algorithm is clearly faster than the competing algorithm in [20]. Referring to Sections 2 and 3, the proposed algorithm uses subspace optimization method to correct the search direction, which could ensure $F\left(l^{i+1}\right) \leq F\left(l^{i+1}\right)$ is valid on the $i$ th iteration. So it is clear that the proposed method is more efficient. The analysis is verified by the experiments results above.

5.2. Comparison Experiment for the $l_{2}-T V$ Model. In the subsection, the algorithm in [18] and ours will be compared. Being different from $[19,20]$, [18] involves a fitting of the auxiliary variable to $D f$ which has superior performance compared to fitting $f[18,23,32]$. The additive noise is selected as Gaussian noise. Here four sets of the standard deviation of noise are chosen: 0.01, 0.05, 0.1, and 0.2. Same methods appearing in [18] will be used to solve the primal alternating direction method.

Similarly, we will first select two cases that have significant improvement of the visual sense to illustrate our method's restoration quality. Figures 3(a) and 4(a) show the original 
TABLE 1: Summary results for the uniform noise.

\begin{tabular}{|c|c|c|c|c|c|c|c|c|}
\hline \multirow{2}{*}{ Image } & \multirow{2}{*}{ Noise } & \multirow{2}{*}{ Blur } & \multicolumn{2}{|c|}{ SNR } & \multicolumn{2}{|c|}{ PSNR } & \multicolumn{2}{|c|}{$\mathrm{CPU}$} \\
\hline & & & {$[20]$} & Ours & {$[20]$} & Ours & {$[20]$} & Ours \\
\hline \multirow{9}{*}{ Camera } & \multirow{3}{*}{$(0,0.05)$} & $5 \times 5$ & 21.25 & 21.46 & 26.83 & 26.84 & 5.49 & 4.84 \\
\hline & & $7 \times 7$ & 19.35 & 19.37 & 24.93 & 24.92 & 6.82 & 5.99 \\
\hline & & $9 \times 9$ & 18.28 & 18.30 & 23.86 & 23.88 & 8.47 & 7.99 \\
\hline & \multirow{3}{*}{$(0,0.1)$} & $5 \times 5$ & 18.12 & 18.13 & 23.70 & 23.73 & 6.12 & 5.93 \\
\hline & & $7 \times 7$ & 17.20 & 17.21 & 22.78 & 22.77 & 8.14 & 7.09 \\
\hline & & $9 \times 9$ & 16.52 & 16.52 & 22.11 & 22.16 & 8.95 & 8.17 \\
\hline & \multirow{3}{*}{$(0,0.2)$} & $5 \times 5$ & 12.86 & 12.86 & 18.45 & 18.57 & 7.57 & 7.07 \\
\hline & & $7 \times 7$ & 12.90 & 12.91 & 18.48 & 18.48 & 7.82 & 7.00 \\
\hline & & $9 \times 9$ & 12.81 & 12.88 & 18.39 & 18.46 & 9.14 & 8.03 \\
\hline \multirow{9}{*}{ Lena } & \multirow{3}{*}{$(0,0.05)$} & $5 \times 5$ & 24.29 & 24.31 & 29.94 & 29.93 & 29.84 & 27.60 \\
\hline & & $7 \times 7$ & 23.42 & 23.46 & 29.07 & 29.11 & 39.13 & 35.62 \\
\hline & & $9 \times 9$ & 22.60 & 22.67 & 28.26 & 28.26 & 44.05 & 37.16 \\
\hline & \multirow{3}{*}{$(0,0.1)$} & $5 \times 5$ & 19.37 & 19.37 & 25.03 & 25.07 & 30.50 & 24.35 \\
\hline & & $7 \times 7$ & 19.18 & 19.15 & 24.84 & 24.84 & 38.24 & 33.65 \\
\hline & & $9 \times 9$ & 18.90 & 18.92 & 24.55 & 24.57 & 45.47 & 40.36 \\
\hline & \multirow{3}{*}{$(0,0.2)$} & $5 \times 5$ & 13.65 & 13.74 & 19.31 & 19.36 & 44.06 & 39.56 \\
\hline & & $7 \times 7$ & 13.58 & 13.63 & 19.24 & 19.33 & 41.28 & 36.33 \\
\hline & & $9 \times 9$ & 13.25 & 13.25 & 18.99 & 18.97 & 45.49 & 40.68 \\
\hline
\end{tabular}

TABLE 2: Summary results for the speckle noise.

\begin{tabular}{|c|c|c|c|c|c|c|c|c|}
\hline \multirow{2}{*}{ Image } & \multirow{2}{*}{ Noise } & \multirow{2}{*}{ Blur } & \multicolumn{2}{|c|}{ SNR } & \multicolumn{2}{|c|}{ PSNR } & \multicolumn{2}{|c|}{$\mathrm{CPU}$} \\
\hline & & & {$[20]$} & Ours & {$[20]$} & Ours & {$[20]$} & Ours \\
\hline \multirow{9}{*}{ Camera } & \multirow{3}{*}{0} & $5 \times 5$ & 22.06 & 22.12 & 27.64 & 27.64 & 5.09 & 4.36 \\
\hline & & $7 \times 7$ & 19.94 & 19.93 & 25.52 & 25.57 & 7.55 & 6.05 \\
\hline & & $9 \times 9$ & 18.72 & 18.74 & 23.89 & 23.90 & 8.13 & 6.88 \\
\hline & \multirow{3}{*}{0.01} & $5 \times 5$ & 20.32 & 20.33 & 25.90 & 25.93 & 5.88 & 5.02 \\
\hline & & $7 \times 7$ & 19.22 & 19.27 & 24.83 & 24.84 & 8.30 & 6.91 \\
\hline & & $9 \times 9$ & 18.28 & 18.28 & 24.30 & 24.35 & 8.13 & 7.04 \\
\hline & \multirow{3}{*}{0.05} & $5 \times 5$ & 15.77 & 15.79 & 21.35 & 21.35 & 9.48 & 8.25 \\
\hline & & $7 \times 7$ & 14.95 & 14.96 & 20.54 & 20.55 & 9.16 & 7.25 \\
\hline & & $9 \times 9$ & 12.63 & 12.62 & 18.21 & 18.24 & 9.87 & 8.23 \\
\hline \multirow{9}{*}{ Lena } & \multirow{3}{*}{0} & $5 \times 5$ & 28.33 & 28.34 & 33.99 & 34.01 & 29.94 & 27.61 \\
\hline & & $7 \times 7$ & 26.07 & 26.10 & 31.72 & 31.72 & 37.44 & 32.56 \\
\hline & & $9 \times 9$ & 24.59 & 24.62 & 30.25 & 30.61 & 45.29 & 38.65 \\
\hline & \multirow{3}{*}{0.01} & $5 \times 5$ & 23.64 & 23.69 & 29.30 & 29.32 & 36.86 & 32.58 \\
\hline & & $7 \times 7$ & 23.86 & 23.94 & 28.92 & 29.00 & 42.32 & 36.32 \\
\hline & & $9 \times 9$ & 23.24 & 23.24 & 28.89 & 28.92 & 50.97 & 43.65 \\
\hline & \multirow{3}{*}{0.05} & $5 \times 5$ & 18.23 & 18.25 & 23.89 & 23.90 & 61.20 & 55.63 \\
\hline & & $7 \times 7$ & 16.58 & 16.57 & 22.24 & 22.31 & 61.31 & 56.86 \\
\hline & & $9 \times 9$ & 16.48 & 16.52 & 20.14 & 20.14 & 71.48 & 63.53 \\
\hline
\end{tabular}

images. Figures 3(b) and 4(b) show the observed images. Figure 3(c) shows the restored Camera image by algorithm in [18]; Figure 3(d) shows the restored Camera image by our method, where the standard deviation of noise is 0.1 and the support is equal to $9 \times 9$. Figure 4 (c) shows the restored Lena image by algorithm in [18]; Figure 4(d) shows the restored Lena image by our method, where the standard deviation of noise is 0.2 and the support is equal to $9 \times 9$. Table 3 shows more image restoration results with the different support and standard deviation of noise.
As in Section 5.1, Figures 3 and 4 show that the quality of restoration images by using our method is better than those restored by the algorithm of [18] in the two cases. We also can know from Table 3 that the SNR and PSNR of the images restored by our method both are higher than the results of [18] in most cases. With the same reasons mentioned in $l_{1}$ TV model, all computational time required by our method is significantly less than that required by the algorithm in [18]. So the performance of the algorithm in [18] also has been improved through the correction. 
TABLE 3: Summary results for Gaussian noise.

\begin{tabular}{|c|c|c|c|c|c|c|c|c|}
\hline \multirow{2}{*}{ Image } & \multirow{2}{*}{ Noise } & \multirow{2}{*}{ Blur } & \multicolumn{2}{|c|}{ SNR } & \multicolumn{2}{|c|}{ PSNR } & \multicolumn{2}{|c|}{$\mathrm{CPU}$} \\
\hline & & & [18] & Ours & [18] & Ours & {$[18]$} & Ours \\
\hline \multirow{12}{*}{ Camera } & \multirow{3}{*}{0.01} & $5 \times 5$ & 21.99 & 21.98 & 27.57 & 27.61 & 2.45 & 2.31 \\
\hline & & $7 \times 7$ & 19.90 & 19.93 & 25.49 & 25.49 & 2.67 & 2.32 \\
\hline & & $9 \times 9$ & 18.69 & 18.69 & 24.27 & 24.28 & 2.73 & 2.63 \\
\hline & \multirow{3}{*}{0.05} & $5 \times 5$ & 20.64 & 20.69 & 26.22 & 26.24 & 1.83 & 1.62 \\
\hline & & $7 \times 7$ & 19.04 & 19.12 & 24.62 & 24.66 & 7.60 & 7.02 \\
\hline & & $9 \times 9$ & 18.01 & 18.07 & 23.59 & 23.59 & 2.57 & 2.26 \\
\hline & \multirow{3}{*}{0.1} & $5 \times 5$ & 17.97 & 17.99 & 23.55 & 23.58 & 1.84 & 1.56 \\
\hline & & $7 \times 7$ & 17.06 & 17.11 & 22.64 & 22.63 & 2.20 & 2.12 \\
\hline & & $9 \times 9$ & 16.41 & 16.40 & 22.00 & 22.61 & 2.22 & 1.98 \\
\hline & \multirow{3}{*}{0.2} & $5 \times 5$ & 13.37 & 17.38 & 18.95 & 18.94 & 1.78 & 1.51 \\
\hline & & $7 \times 7$ & 13.11 & 13.10 & 18.70 & 18.73 & 1.68 & 1.53 \\
\hline & & $9 \times 9$ & 12.91 & 12.93 & 18.49 & 18.61 & 1.86 & 1.68 \\
\hline \multirow{12}{*}{ Lena } & \multirow{3}{*}{0.01} & $5 \times 5$ & 28.05 & 28.10 & 33.71 & 33.73 & 11.89 & 10.32 \\
\hline & & $7 \times 7$ & 25.90 & 25.91 & 31.56 & 31.62 & 12.93 & 10.33 \\
\hline & & $9 \times 9$ & 24.47 & 24.49 & 30.13 & 30.15 & 13.32 & 10.92 \\
\hline & \multirow{3}{*}{0.05} & $5 \times 5$ & 24.09 & 24.06 & 29.74 & 29.77 & 10.39 & 9.66 \\
\hline & & $7 \times 7$ & 23.12 & 23.12 & 28.78 & 28.79 & 11.26 & 9.65 \\
\hline & & $9 \times 9$ & 22.33 & 22.42 & 27.99 & 28.12 & 13.18 & 11.56 \\
\hline & \multirow{3}{*}{0.1} & $5 \times 5$ & 19.50 & 19.55 & 25.16 & 25.17 & 9.14 & 8.68 \\
\hline & & $7 \times 7$ & 19.19 & 19.23 & 24.85 & 24.84 & 10.26 & 9.33 \\
\hline & & $9 \times 9$ & 18.86 & 18.88 & 24.52 & 24.51 & 11.79 & 9.91 \\
\hline & \multirow{3}{*}{0.2} & $5 \times 5$ & 13.81 & 13.81 & 19.47 & 19.44 & 7.14 & 6.56 \\
\hline & & $7 \times 7$ & 13.83 & 13.86 & 19.49 & 19.83 & 8.47 & 7.82 \\
\hline & & $9 \times 9$ & 13.78 & 14.01 & 19.44 & 19.87 & 9.78 & 7.97 \\
\hline
\end{tabular}

\section{Concluding Remarks}

Taking the $l_{1}$ TV model in image restoration as an example, a modified method is proposed to overcome drawbacks of the primal alternating direction method in this paper. We have illustrated that our method about how to correct the search direction improves the optimization performance. In addition, the convergence of the primal alternating direction method has been proven under some weaker conditions, and thus the convergence of proposed method is easily obtained by the equivalence between them. The experimental results based on two models in $[18,20]$ show that the proposed method could enhance the quality of restored images in most cases and the efficiency of algorithms has been significantly improved. In fact, our method can be applied to many other cases optimized alternatively.

\section{Conflicts of Interest}

The authors declare that there are no conflicts of interest regarding the publication of this paper.

\section{Acknowledgments}

This work is supported by the Fundamental Research Funds for the Central Universities of Southwest University for Nationalities (no. 2015NZYQN30), the Key Fund Project of
Sichuan Provincial Department of Education (no. 17ZA0414), and the National Science Foundation of China (no. 61273311). The authors are grateful to the author, Xiaoxia Guo, of [20] for providing programmes.

\section{References}

[1] A. K. Katsaggelos, Digital Image Restoration, Springer-Verlag, Berlin, Germany, 1991.

[2] A. S. Carasso, "Linear and nonlinear image deblurring: a documented study," SIAM Journal on Numerical Analysis, vol. 36, no. 6, pp. 1659-1689, 1999.

[3] R. C. Gonzalez and R. E. Woods, Digital Image Processing, Prentice Hall, Upper Saddle River, NJ, USA, 2nd edition, 2002.

[4] M. K. Ng, R. H. Chan, and W.-C. Tang, "A fast algorithm for deblurring models with Neumann boundary conditions," SIAM Journal on Scientific Computing, vol. 21, no. 3, pp. 851-866, 1999.

[5] A. M. Bruckstein, D. L. Donoho, and M. Elad, "From sparse solutions of systems of equations to sparse modeling of signals and images," SIAM Review, vol. 51, no. 1, pp. 34-81, 2009.

[6] M. J. Black and A. Rangarajan, "On the unification of line processes, outlier rejection, and robust statistics with applications in early vision," International Journal of Computer Vision, vol. 19, no. 1, pp. 57-91, 1996.

[7] A. N. Tikhonov and V. Y. Arsenin, "Solutions of Ill-Posed Problem," Mathematics of Computation, vol. 23, 491 pages, 1977. 
[8] J. Liu, T.-Z. Huang, I. W. Selesnick, X.-G. Lv, and P.-Y. Chen, "Image restoration using total variation with overlapping group sparsity," Information Sciences, vol. 295, pp. 232-246, 2015.

[9] L. I. Rudin and S. Osher, "Total variation based image restoration with free local constraints," in Proceedings of the 1st IEEE International Conference on Image Processing, vol. 1, pp. 31-35, IEEE, Austin, Tex, USA, November 1994.

[10] A. Langer, "Automated parameter selection for total variation minimization in image restoration," Journal of Mathematical Imaging and Vision, vol. 57, no. 2, pp. 239-268, 2017.

[11] X. Liu, "Augmented Lagrangian method for total generalized variation based Poissonian image restoration," Computers \& Mathematics with Applications. An International Journal, vol. 71, no. 8, pp. 1694-1705, 2016.

[12] J. Liu, T.-Z. Huang, X.-G. Lv, and S. Wang, "High-order total variation-based Poissonian image deconvolution with spatially adapted regularization parameter," Applied Mathematical Modelling, vol. 45, pp. 516-529, 2017.

[13] A. Chambolle, "An algorithm for total variation minimization and applications," Journal of Mathematical Imaging and Vision, vol. 20, no. 1-2, pp. 89-97, 2004.

[14] T. F. Chan and K. Chen, "An optimization-based multilevel algorithm for total variation image denoising," Multiscale Modeling \& Simulation. A SIAM Interdisciplinary Journal, vol. 5, no. 2, pp. 615-645, 2006.

[15] M. K. Ng, L. Qi, Y.-F. Yang, and Y.-M. Huang, "On semismooth Newton's methods for total variation minimization," Journal of Mathematical Imaging and Vision, vol. 27, no. 3, pp. 265-276, 2007.

[16] T. F. Chan, G. H. Golub, and P. Mulet, "A nonlinear primaldual method for total variation-based image restoration," SIAM Journal on Scientific Computing, vol. 20, no. 6, pp. 1964-1977, 1999.

[17] M. Hintermuller and K. Kunisch, "Total bounded variation regularization as a bilaterally constrained optimization problem," SIAM Journal on Applied Mathematics, vol. 64, no. 4, pp. 13111333, 2004.

[18] Y. Huang, M. K. Ng, and Y.-W. Wen, "A fast total variation minimization method for image restoration," Multiscale Modeling \& Simulation. A SIAM Interdisciplinary Journal, vol. 7, no. 2, pp. 774-795, 2008.

[19] Y. Wang, J. Yang, W. Yin, and Y. Zhang, "A new alternating minimization algorithm for total variation image reconstruction," SIAM Journal on Imaging Sciences, vol. 1, no. 3, pp. 248-272, 2008.

[20] X. Guo, F. Li, and M. K. Ng, "A fast l1-TV algorithm for image restoration," SIAM Journal on Scientific Computing, vol. 31, no. 3, pp. 2322-2341, 2009.

[21] Z. Zhi, Y. Sun, and Z.-F. Pang, "Two-stage image segmentation scheme based on inexact alternating direction method," Numerical Mathematics: Theory, Methods and Applications, vol. 9, no. 3, pp. 451-469, 2016.

[22] F. Wang, X.-L. Zhao, and M. K. Ng, "Multiplicative noise and blur removal by framelet decomposition and l1-based L-curve method," IEEE Transactions on Image Processing, vol. 25, no. 9, pp. 4222-4232, 2016.

[23] J. Yang, W. Yin, Y. Zhang, and Y. Wang, "A fast algorithm for edge-preserving variational multichannel image restoration," SIAM Journal on Imaging Sciences, vol. 2, no. 2, pp. 569-592, 2009.
[24] W. Y. Sun and Y. X. Yuan, Optimization Theory and Method: Nonlinear Programming, Springer-Verlag, Berlin, Germany, 2006.

[25] S. Boyd and L. Vandenberghe, Convex Optimization, Cambridge University Press, Cambridge, UK, 2004.

[26] S. Boyd, N. Parikh, E. Chu, B. Peleato, and J. Eckstein, "Distributed optimization and statistical learning via the alternating direction method of multipliers," Foundations and Trends in Machine Learning, vol. 3, no. 1, pp. 1-122, 2010.

[27] Z.-J. Bai, D. Cassani, M. Donatelli, and S. Serra-Capizzano, "A fast alternating minimization algorithm for total variation deblurring without boundary artifacts," Journal of Mathematical Analysis and Applications, vol. 415, no. 1, pp. 373-393, 2014.

[28] M. Elad, B. Matalon, and M. Zibulevsky, "Coordinate and subspace optimization methods for linear least squares with non-quadratic regularization," Applied and Computational Harmonic Analysis, vol. 23, no. 3, pp. 346-367, 2007.

[29] P. L. Combettes and V. R. Wajs, "Signal recovery by proximal forward-backward splitting," Multiscale Modeling \& Simulation. A SIAM Interdisciplinary Journal, vol. 4, no. 4, pp. 1168-1200, 2005.

[30] D. P. Bertsekas, A. Nedic, and A. E. Ozdaglar, Convex Analysis and Optimization, Athena Scientific, Belmont, Mass, USA, 2003.

[31] D. M. Young, Iterative Solution of Large Linear Systems, Academic Press, NY, USA, 1971.

[32] M. Nikolova, M. K. Ng, and C.-P. Tam, "Fast nonconvex nonsmooth minimization methods for image restoration and reconstruction," IEEE Transactions on Image Processing, vol. 19, no. 12, pp. 3073-3088, 2010. 


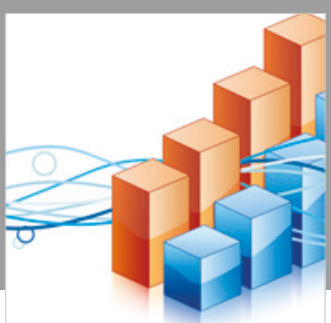

Advances in

Operations Research

\section{-n-m}
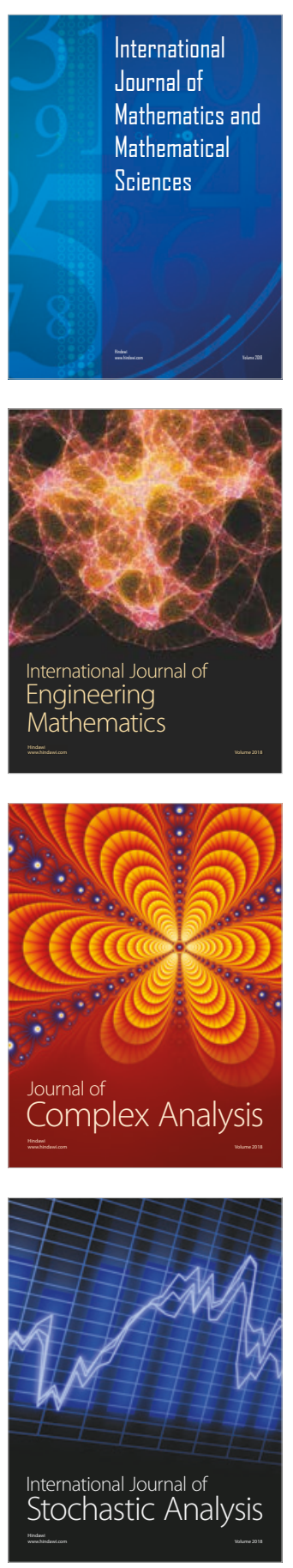
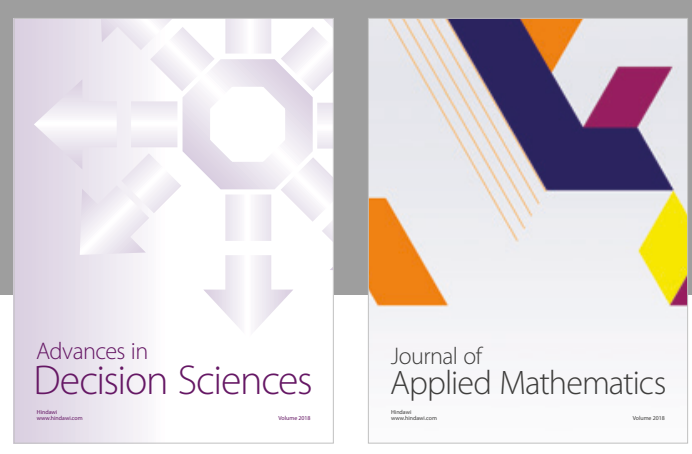

Journal of

Applied Mathematics
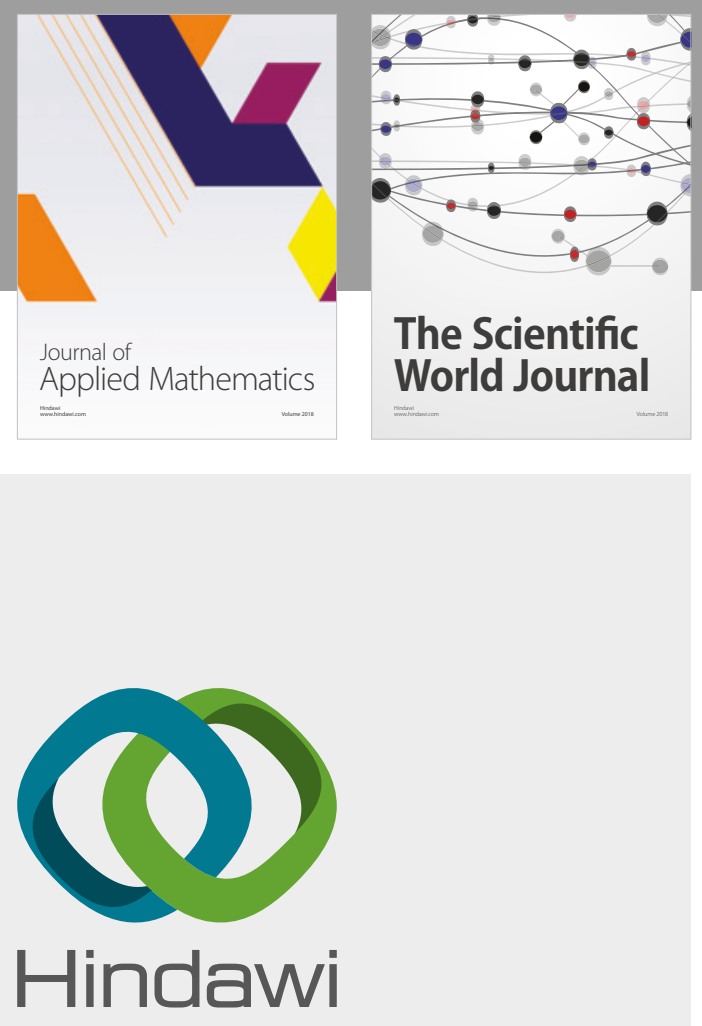

Submit your manuscripts at

www.hindawi.com

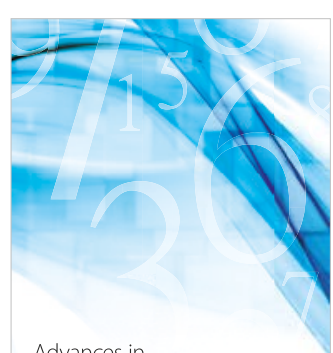

Advances in
Numerical Analysis
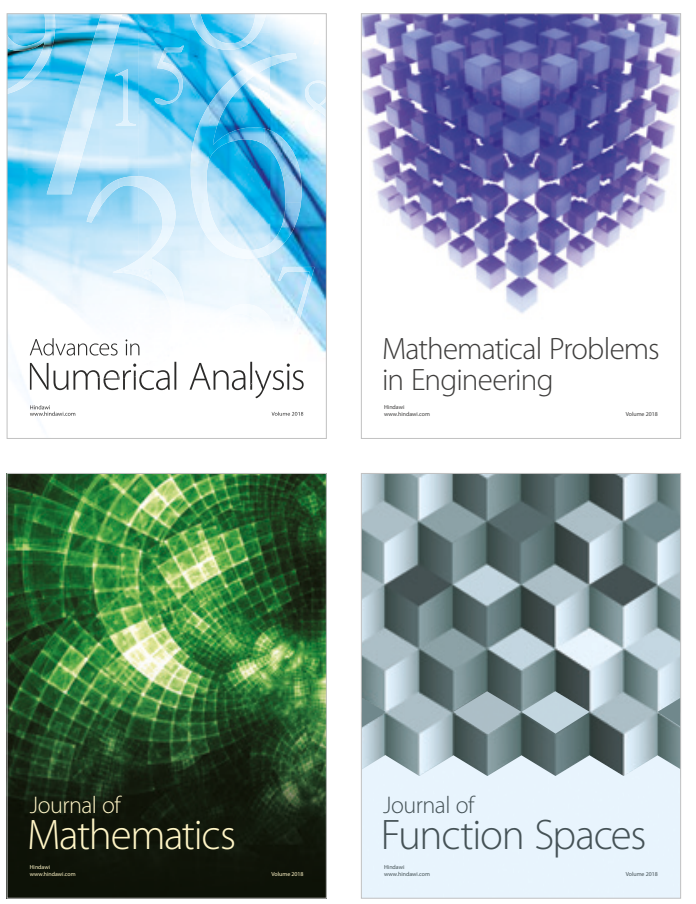

Mathematical Problems in Engineering

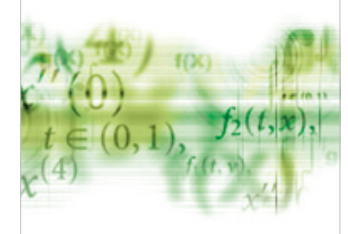

International Journal of

Differential Equations

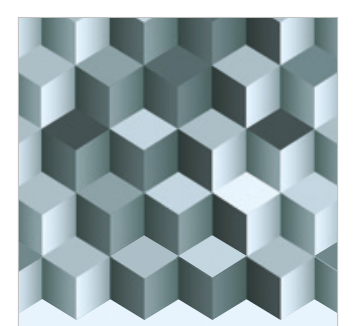

Journal of

Function Spaces

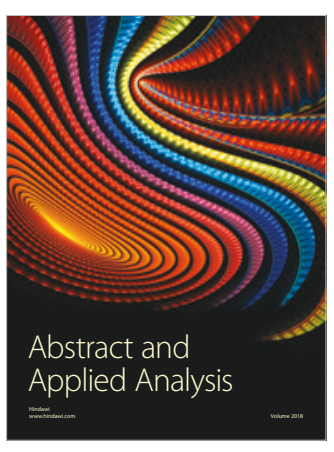

The Scientific

World Journal

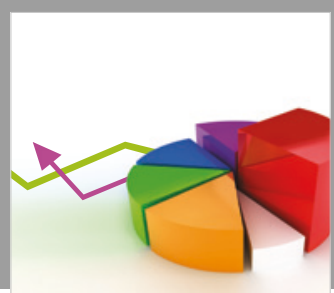

Journal of

Probability and Statistics
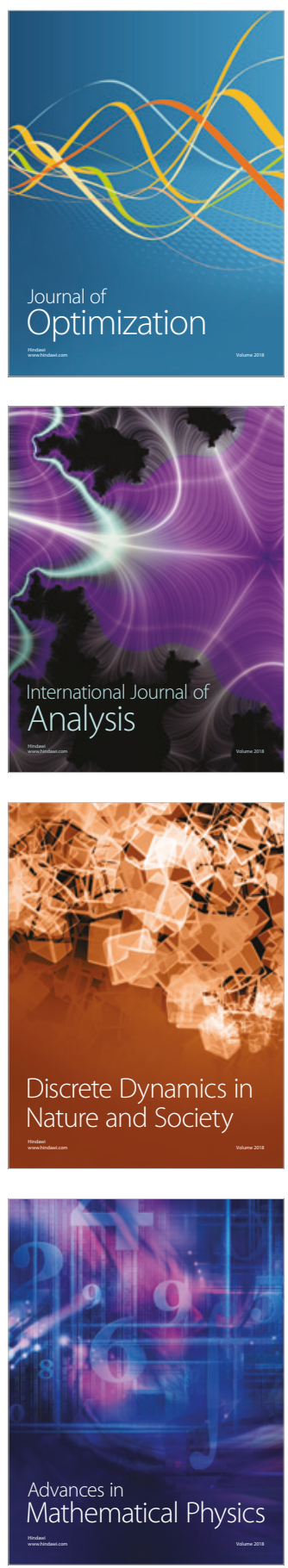